\title{
An Approximate Analytical Approach to Investigate the Stability and Response of Holonomic Problems in Classic Dynamics by Means of the Homotopy Perturbation Method
}

\author{
Amin Kimiaeifar \\ Department of Mechanical and Manufacturing Engineering, Aalborg University, \\ Pontoppidanstraede 101, DK-9220 Aalborg East, Denmark \\ Reprint requests to A. K.; E-mail: a.kimiaeifar@gmail.com \\ Z. Naturforsch. 66a, 461 - 467 (2011) / DOI: 10.5560/ZNA.2011-0001 \\ Received July 29, 2010 / revised March 30, 2011 \\ In this work, a powerful analytical method called homotopy perturbation method (HPM) is used \\ to obtain the series solution for nonlinear problems in classic dynamics. The governing equations are \\ obtained using the Lagrange method and solved analytically by HPM. The present solution gives an \\ expression which is valid for the whole domain of the solution. Comparisons of the obtained solutions \\ with numerical results indicate that HPM is effective and convenient for solving holonomic problems \\ and can be used in the same way for other nonlinear problems.
}

Key words: Homotopy Perturbation Method; Lagrange Method; Nonlinear Dynamics; Phase Plane.

\section{Introduction}

Nonlinear phenomena play important roles in applied mathematics, physics, and also in engineering problems in which the variation of each parameter depends on different factors. Solving nonlinear equations may guide authors to have a better understanding of the problems physics common observations. Moreover, obtaining exact solutions for nonlinear problems is a great purpose which has been quite untouched.

In addition, in recent years, scientists have presented some new methods for solving nonlinear partial differential equations; for instance, the Bäcklund transformation method [1], the Lie group method [2], Adomian's decomposition method [3], the inverse scattering method [4], the homotopy analysis method [5, 6], and He's homotopy perturbation method and parameter expanding method [7-14].

HPM is one of the most effective and convenient methods for both linear and nonlinear equations. This method does not depend on a small/large parameter. HPM has been shown to effectively, easily, and accurately solve a large class of linear and nonlinear problems with components converging rapidly to accurate solutions.

In this study, first by using the Lagrange method, the governing equations are obtained then HPM is ap- plied to find an analytical solution for the nonlinear differential equation governing nonlinear problems in dynamics and is used to investigate the behaviour of the limit cycle and phase plane. The perturbation method is not able to solve this problem because there is no small/large parameter. The numerical solution based on the shooting method and the fourth-order RungeKutta method developed by the author to indicate the accuracy of the results. It is shown that the results are in very good agreement with those obtained using numerical methods. Finally, the influence of constant parameters on the system response and its stability is shown.

\section{Lagrange Equations}

A brief development of the Lagrange equation is shown, presented here for the general form of kinetic and potential energies equations.

It is clear that based on a conservative system, the total energy should be zero:

$$
\mathrm{d}(T+U)=0
$$

The potential energy $U$ is a function only of generalized velocities, $\dot{q}_{i}$, and the kinetic energy $T$ is a function of the generalized coordinates $q_{i}$ and $\dot{q}_{i}$ which can 
be shown as follows:

$$
\begin{aligned}
T & =T\left(q_{1}, q_{2}, \ldots q_{n}, \dot{q}_{1}, \dot{q}_{2}, \ldots \dot{q}_{n}\right), \\
U & =U\left(\dot{q}_{1}, \dot{q}_{2}, \ldots \dot{q}_{n}\right) .
\end{aligned}
$$

The derivative of $T$ with respect to generalized coordinates is

$$
\mathrm{d} T=\sum_{i=1}^{N} \frac{\partial T}{\partial q_{i}} \mathrm{~d} q_{i}+\sum_{i=1}^{N} \frac{\partial T}{\partial \dot{q}_{i}} \mathrm{~d} \dot{q}_{i} .
$$

To eliminate the second term with $\mathrm{d} \dot{q}_{i}$, we start with the equation for the kinetic energy:

$$
T=\frac{1}{2} \sum_{i=1}^{N} \sum_{j=1}^{N} m_{i j} \dot{q}_{i} \dot{q}_{j}
$$

Differentiating this equation with respect to $\dot{q}_{i}$, multiplying by $\dot{q}_{i}$, and summing over $i$ from 1 to $N$, yields the following result:

$$
2 T=\frac{1}{2} \sum_{i=1}^{N} \sum_{j=1}^{N} m_{i j} \dot{q}_{i} \dot{q}_{j}=\sum_{i=1}^{N} \frac{\partial T}{\partial \dot{q}_{j}} \dot{q}_{i}
$$

or

$$
2 T=\sum_{i=1}^{N} \frac{\partial T}{\partial \dot{q}_{j}} \dot{q}_{i}
$$

From the differential of $2 T$ in (7) by using the product rule in calculus, we get

$$
2 \mathrm{~d} T=\sum_{i=1}^{N} \mathrm{~d}\left(\frac{\partial T}{\partial \dot{q}_{i}}\right) \dot{q}_{i}+\sum_{i=1}^{N} \frac{\partial T}{\partial \dot{q}_{i}} \mathrm{~d} \dot{q}_{i} .
$$

And finally for the kinetic energy it is obtained:

$$
\mathrm{d} T=\sum_{i=1}^{N}\left[\frac{\mathrm{d}}{\mathrm{d} T}\left(\frac{\partial T}{\partial \dot{q}_{i}}\right)-\frac{\partial T}{\partial \dot{q}_{i}}\right] \mathrm{d} \dot{q}_{i} .
$$

By doing the same procedure for the potential energy and substituting in (1) results in

$\mathrm{d}(T+U)=\sum_{i=1}^{N}\left[\frac{\mathrm{d}}{\mathrm{d} T}\left(\frac{\partial T}{\partial \dot{q}_{i}}\right)-\frac{\partial T}{\partial \dot{q}_{i}}+\frac{\partial U}{\partial \dot{q}_{i}}\right] \mathrm{d} \dot{q}_{i}=0$.

By doing some simplifications and since $\frac{\partial U}{\partial \dot{q}_{i}}=0,(10)$ can be written as

$$
\frac{\mathrm{d}}{\mathrm{d} t}\left(\frac{\partial L}{\partial \dot{q}_{i}}\right)-\frac{\partial L}{\partial \dot{q}_{i}}=0, i=1,2, \ldots N .
$$

A. Kimiaeifar - Stability and Response of Holonomic Problems

\section{Basic Idea of HPM}

To illustrate the basic ideas of this method, the following nonlinear general differential equation is considered:

$$
A(u)-f(r)=0, r \in \Omega,
$$

with the boundary conditions as below:

$$
B\left(u, \frac{\partial u}{\partial n}\right)=0, r \in \Gamma
$$

Where $A$ is a general differential operator, $B$ a boundary operator, $f(r)$ a known analytical function, and $\Gamma$ is the boundary of the domain $\Omega$.

The operator $A$ is divided into two parts $L$ (linear part) and $N$ (nonlinear part). Therefor (5) results in

$$
L(u)+N(u)-f(r)=0 .
$$

The homotopy-perturbation structure is written as:

$H(v, p)=(1-p)\left[L(v)-L\left(u_{0}\right)\right]+p[A(v)-f(r)]=0$,

where

$$
v(r, p): \Omega \times[0,1] \rightarrow R
$$

Obviously, considering (15) results in

$$
\begin{aligned}
& H(v, 0)=L(v)-L\left(u_{0}\right)=0, \\
& H(v, 1)=A(v)-f(r)=0,
\end{aligned}
$$

where $p \in[0,1]$ is an embedding parameter and $u_{0}$ is the first approximation that satisfies the boundary condition. The process of the changes in $p$ from zero to unity is that of $v(t, p)$ changing from $u_{0}$ to $u_{r}$. By considering $v$ as

$$
v(t)=v_{0}(r)+p v_{1}(r)+p^{2} v_{2}(r)+\ldots,
$$

the best approximation for the solution is

$$
u=\lim _{p \rightarrow 1} v=v_{0}+v_{1}+v_{2}+\ldots
$$

\section{Example 1: A Rotating Mass with Central Force}

As a first example, the motion of a particle in a plane under influence of a central force is considered. In po- 
lar coordinates the motion of a particle $m$ is governed by

$$
\begin{gathered}
\left(m \frac{\mathrm{d}^{2} r(t)}{\mathrm{d} t^{2}}-r{\frac{\mathrm{d} \theta(t)^{2}}{\mathrm{~d} t}}^{2}\right)+F(r)=0, \\
r(0)=a_{1},\left.\quad \frac{\mathrm{d} r(t)}{\mathrm{d} t}\right|_{t=0},=a_{2}, \\
\left(m r \frac{\mathrm{d}^{2} \theta(t)}{\mathrm{d} t^{2}}+2 \frac{\mathrm{d} r(t)}{\mathrm{d} t} \frac{\mathrm{d} \theta(t)}{\mathrm{d} t}\right)=0, \\
\theta(0)=a_{3},\left.\frac{\mathrm{d} \theta(t)}{\mathrm{d} t}\right|_{t=0},=a_{4} .
\end{gathered}
$$

Here, $F(r)$, as external force, is considered as $\sin (r(t))$ and compared with the numerical method. By using Taylor's series expansion for $\sin (r(t))$, the above equation reduces to

$$
\begin{gathered}
\left(m \frac{\mathrm{d}^{2} r(t)}{\mathrm{d} t^{2}}-r \frac{\mathrm{d} \theta(t)^{2}}{\mathrm{~d} t}\right)+\left(r(t)-\frac{1}{6} r^{3}(t)\right)=0, \\
r(0)=\alpha,\left.\frac{\mathrm{d} r(t)}{\mathrm{d} t}\right|_{t=0},=0, \\
\left(m r \frac{\mathrm{d}^{2} \theta(t)}{\mathrm{d} t^{2}}+2 \frac{\mathrm{d} r(t)}{\mathrm{d} t} \frac{\mathrm{d} \theta(t)}{\mathrm{d} t}\right)=0, \\
\theta(0)=\beta,\left.\frac{\mathrm{d} \theta(t)}{\mathrm{d} t}\right|_{t=0},=0 .
\end{gathered}
$$

As it was explained in Part 3, choosing a linear operator is very important, and linear operators must be chosen in such a way that the answer should be helpful for the solution procedures and convergence [12]. According to the HPM, the homotopy construction for (20) can be written as follows:

$$
\begin{aligned}
& (1-p)\left[(\ddot{v}+v)-\frac{\varepsilon}{m}\left(\ddot{r}_{0}+r_{0}\right)\right] \\
& +p\left[m\left(\frac{\mathrm{d}^{2} v(t)}{\mathrm{d} t^{2}}-r \frac{\mathrm{d} u(t)^{2}}{\mathrm{~d} t}\right)+\left(v(t)-\frac{1}{6} v^{3}(t)\right)\right]=0 \\
& (1-p)\left[(u)-\left(\ddot{\theta}_{0}\right)\right] \\
& +p\left[m\left(r \frac{\mathrm{d}^{2} u(t)}{\mathrm{d} t^{2}}+2 \frac{\mathrm{d} v(t)}{\mathrm{d} t} \frac{\mathrm{d} u(t)}{\mathrm{d} t}\right)\right]=0
\end{aligned}
$$

where

$$
\begin{aligned}
& v(t)=v_{0}(t)+p v_{1}(t)+p^{2} v_{2}(t)+\ldots, \\
& u(t)=u_{0}(t)+p u_{1}(t)+p^{2} u_{2}(t)+\ldots,
\end{aligned}
$$

and

$$
\begin{aligned}
& r(t)=\lim _{p \rightarrow 1} v(t)=v_{0}(t)+v_{1}(t)+v_{2}(t)+\ldots, \\
& \theta(t)=\lim _{p \rightarrow 1} u(t)=u_{0}(t)+u_{1}(t)+u_{2}(t)+\ldots .
\end{aligned}
$$

It should be noted that $\varepsilon$ is an unknown parameter which further is determined. The initial boundary conditions are as follows:

$$
\begin{aligned}
& v_{0}(0)=r_{0}(0)=\alpha, \quad \dot{v}_{0}(0)=\dot{r}_{0}(0)=0, \\
& u_{0}(0)=\theta_{0}(0)=\beta, \quad \dot{u}_{0}(0)=\dot{\theta}_{0}(0)=0 .
\end{aligned}
$$

Substituting (22) and (23) into (21) and rearranging based on powers of the $p$-terms, then solving the obtained linear differential equations yield following answer:

$$
\begin{aligned}
& v_{0}(t)=\alpha \cos \left(\frac{\sqrt{\varepsilon} t}{\sqrt{m}}\right), \\
& u_{0}(t)=\beta,
\end{aligned}
$$

$$
u_{1}(t)=0 \text {. }
$$

To prevent divergence, pointed out by $\mathrm{He}$ [12], $\varepsilon$ should be determined in such away that secular terms vanish. The secular terms are known as expressions such as $t \sin t, t \cos t$. Hereby, $\varepsilon$ is calculated as

$$
\varepsilon=1-\frac{1}{8} \alpha^{2} \text {. }
$$




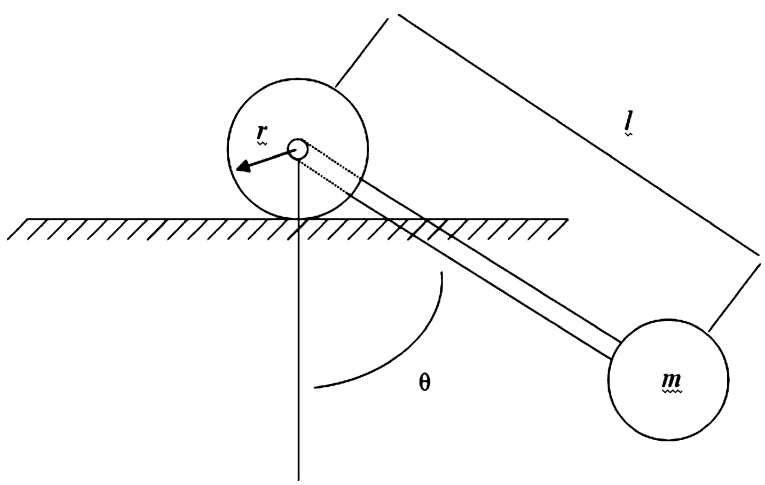

Fig. 1. Geometry of Example 2.

As it was mentioned, the solution of (20) for $p \rightarrow 1$ will be as follows:

$$
\begin{aligned}
& r(t)=v_{0}(t)+v_{1}(t), \\
& \theta(t)=u_{0}(t)+u_{1}(t) .
\end{aligned}
$$

\section{Example 2}

A rigid rod is rigidly attached to the axle as shown in Figure 1. The wheels roll without slip as the pendulum swings back and forth. Only the ball on the end of the pendulum has appreciable mass, and it may be considered as particle.

By using the Lagrange method, the governing equation can be expressed as follows:

$\left(l^{2}+r^{2}-2 r l \cos \theta\right) \ddot{\theta}+r l \sin \theta \dot{\theta}^{2}+g l \sin \theta=0$.

Substitute the two first terms of the Taylor series instead of $\sin \theta$ and $\cos \theta$, the above equation reduces
A. Kimiaeifar - Stability and Response of Holonomic Problems

to

$$
\begin{aligned}
& \left(l^{2}+r^{2}-2 r l\left(1-\frac{\theta^{2}}{2}\right)\right) \ddot{\theta} \\
& +r l\left(\theta-\frac{\theta^{3}}{6}\right) \dot{\theta}^{2}+g l\left(\theta-\frac{\theta^{3}}{6}\right)=0 .
\end{aligned}
$$

The initial boundary conditions are considered as

$$
\theta(0)=1, \dot{\theta}(0)=0
$$

According to the HPM, the homotopy construction for (36) can be written as follows:

$$
\begin{aligned}
& (1-p)\left[(\ddot{v}+v)-\varepsilon\left(\ddot{\theta}_{0}+\theta_{0}\right)\right] \\
& +p\left[\left(l^{2}+r^{2}-2 r l\left(1-\frac{\theta^{2}}{2}\right)\right) \ddot{\theta}\right. \\
& \left.+r l\left(\theta-\frac{\theta^{3}}{6}\right) \dot{\theta}^{2}+g l\left(\theta-\frac{\theta^{3}}{6}\right)=0\right]=0,
\end{aligned}
$$

where

$$
v(t)=v_{0}(t)+p v_{1}(t)+p^{2} v_{2}(t)+\ldots
$$

and

$$
\theta(t)=\lim _{p \rightarrow 1} v(t)=v_{0}(t)+v_{1}(t)+v_{2}(t)+\ldots
$$

The initial boundary conditions are

$$
v_{0}(0)=\theta_{0}(0)=1, \quad \dot{v}_{0}(0)=\dot{\theta}_{0}(0)=0 .
$$

Substituting (39) and (40) into (38) and rearranging based on powers of the $p$-terms, then solving the obtained linear differential equations and also vanishing the secular term one obtains:

$$
v_{0}(t)=\cos (\sqrt{\varepsilon} t),
$$

$$
\begin{aligned}
v_{1}(t)= & \frac{m\left(144 l^{2} \varepsilon-248 r l \varepsilon-129 g l+144 r^{2} \varepsilon\right) \cos (\sqrt{\varepsilon} t)}{576 \varepsilon}+\frac{1}{2 \varepsilon^{3 / 2}}\left[-\frac{47}{384} l\left(r \varepsilon^{3 / 2}+\frac{4}{47} g \sqrt{\varepsilon}\right) \cos (3 \sqrt{\varepsilon} t)\right. \\
& +\frac{1}{1152} r l \cos (5 \sqrt{\varepsilon} t) \varepsilon^{3 / 2}+\left(\left(\frac{1}{2} r^{2}-\frac{71}{96} r l+\frac{1}{2} l^{2}\right) \varepsilon^{3 / 2}-\frac{7}{16} l \sqrt{\varepsilon} g\right) \cos (\sqrt{\varepsilon} t) \\
& \left.\cdot t \varepsilon \sin (3 \sqrt{\varepsilon} t)\left(l^{2} \varepsilon+\left(\frac{-71}{48} r \varepsilon-\frac{7}{8} g\right) l+r^{2} \varepsilon\right)\right]
\end{aligned}
$$


where

$$
\varepsilon=\frac{42 g l}{48 l^{2}-71 r l+48 r^{2}} .
$$

Finally the answer is

$$
\theta(t)=v_{0}(t)+v_{1}(t)
$$

\section{Results and Discussion}

In this paper, the effectiveness of the presented homotopy perturbation method was investigated by

(a)

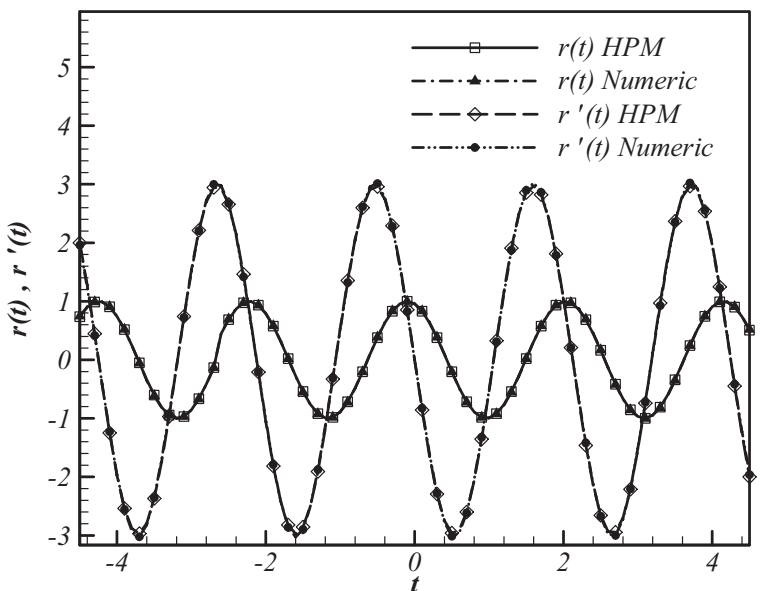

(b)

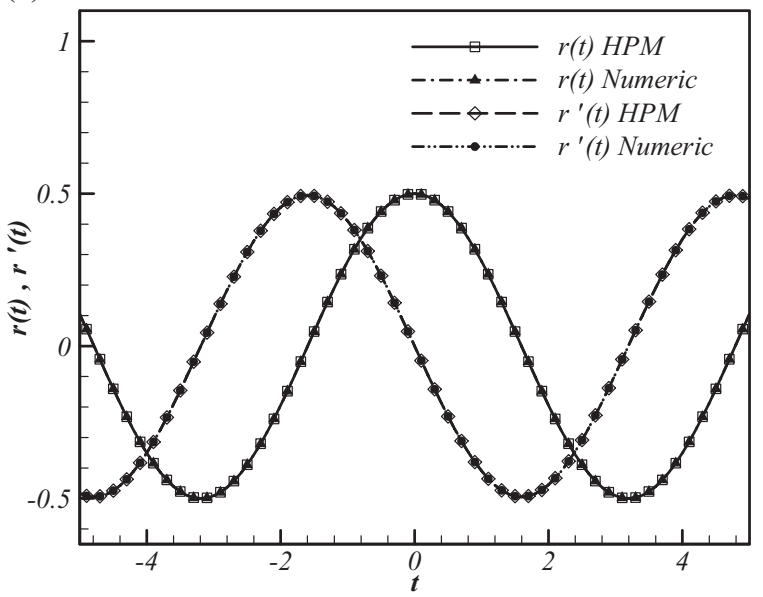

Fig. 2. Effects of constant parameters on position and velocity, Example 1; (a): $m=0.1, \alpha=1.0, \beta=1.0$; (b): $m=$ $1.0, \alpha=0.5, \beta=1.0$. considering two nonlinear dynamics problems. To validate the HPM results, convergence studies were carried out and the results were compared with those obtained using numerical methods which is shown in Figures 2, 3, and Table 1. It is clear that the maximum relative error (R.E.) is about 0.005 which from the view point of engineering is quiet acceptable. In addition to investigate the behaviour of each system from the view point of stability, after convincing of the solution, the phase planes have been plotted and are shown in Figures 4 and 5. By using HPM, the influence of constant physical parameters on the stability is easy to investigate and there is no need to solve the problem by any variation in an input variable of such numerical methods.

(a)

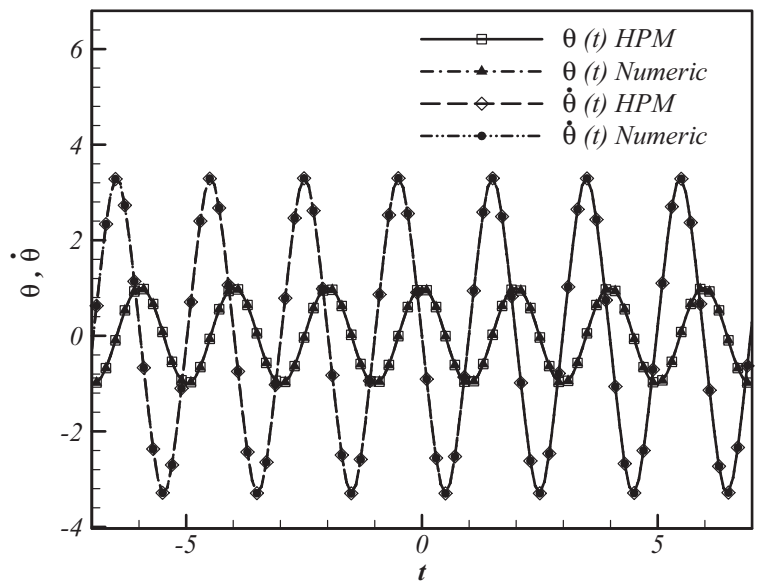

(b)

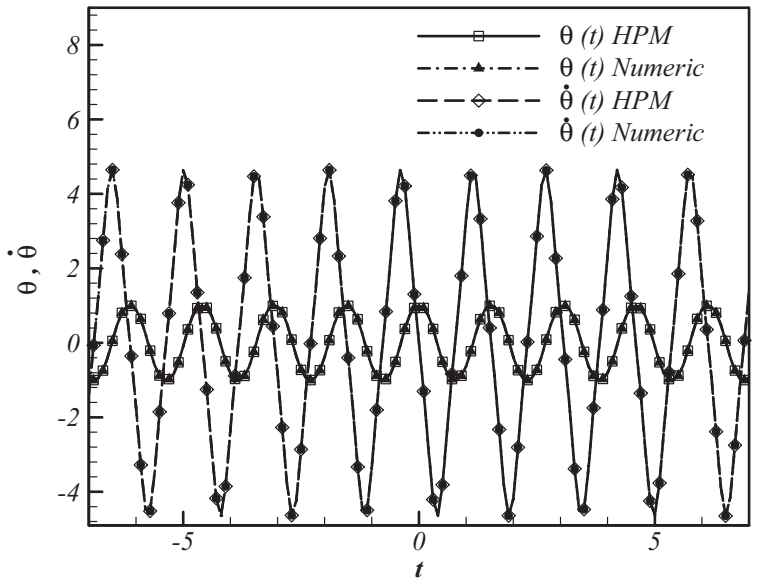

Fig. 3. Effects of constant parameters on position and velocity, Example 2; (a): $l=1.0, r=0.1$; (b): $l=1.0, r=0.5$. 
A. Kimiaeifar · Stability and Response of Holonomic Problems

Table 1. Comparing the present analytical and numerical results for Example 1; $\alpha=0.5, m=0.5$.

\begin{tabular}{lrrrrrr}
\hline & \multicolumn{5}{c}{ R.E. $=\left|\frac{r_{\text {Numeric }}-r_{\text {HPM }}}{r_{\text {Numeric }}}\right|$} & \\
$t$ & \multicolumn{1}{c}{ HPM } & Numeric & R.E. & HPM & Numeric & R.E. \\
\hline 0.1 & 0.49519 & 0.49522 & 0.00005 & -0.09606 & -0.09555 & 0.00528 \\
0.2 & 0.48085 & 0.48095 & 0.00020 & -0.19035 & -0.18943 & 0.00483 \\
0.3 & 0.45744 & 0.45724 & 0.00044 & -0.27996 & -0.28111 & 0.00409 \\
0.4 & 0.42480 & 0.42512 & 0.00075 & -0.36664 & -0.36548 & 0.00318 \\
0.5 & 0.38414 & 0.38457 & 0.00111 & -0.44529 & -0.44435 & 0.00212 \\
1.0 & 0.08918 & 0.08943 & 0.00276 & -0.68653 & -0.68819 & 0.00240 \\
2.0 & -0.46850 & -0.46865 & 0.00031 & -0.24261 & -0.24158 & 0.00426 \\
5.0 & 0.39029 & 0.39075 & 0.00117 & -0.43477 & -0.43371 & 0.00244 \\
10 & 0.10830 & 0.10872 & 0.00392 & -0.68111 & -0.68262 & 0.00222 \\
20 & -0.45354 & -0.45363 & 0.00020 & -0.29246 & -0.29168 & 0.00266 \\
\hline
\end{tabular}

(a)

(a)

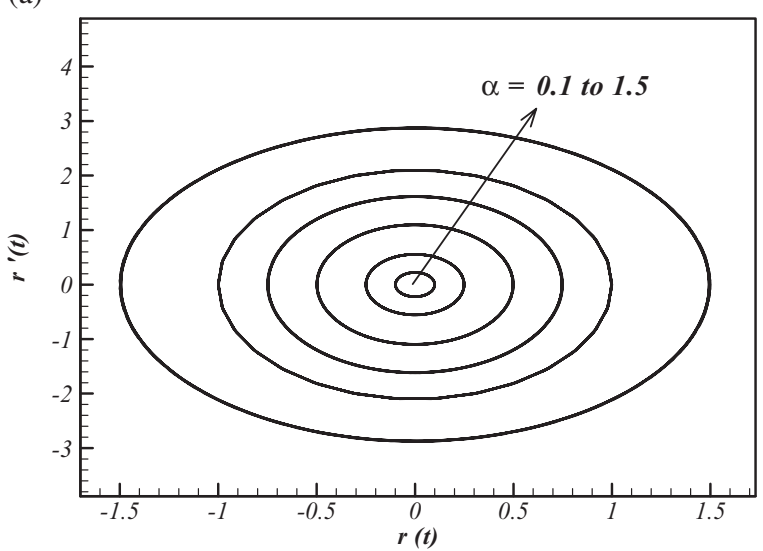

(b)

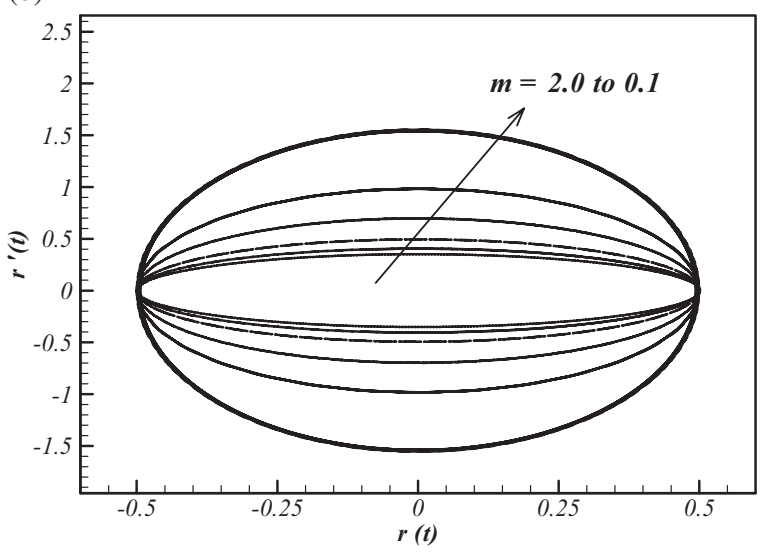

Fig. 4. Phase plane for Example 1; (a): variation of $\alpha$; (b): variation of $m$

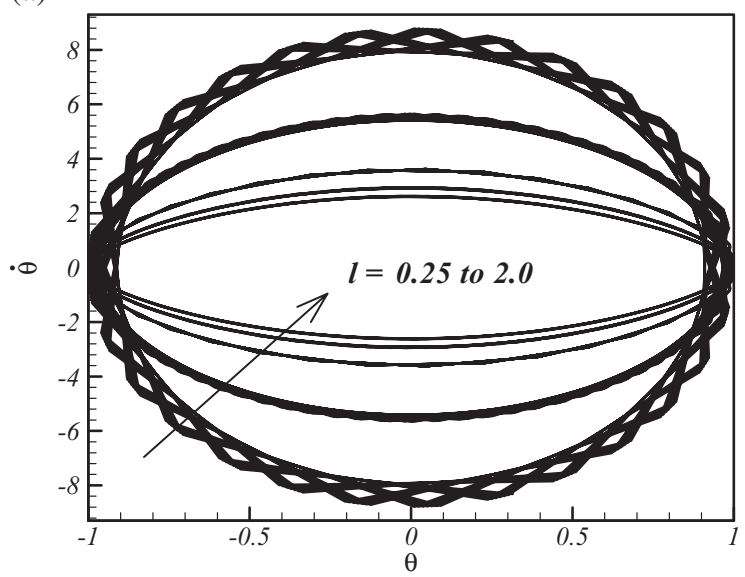

(b)

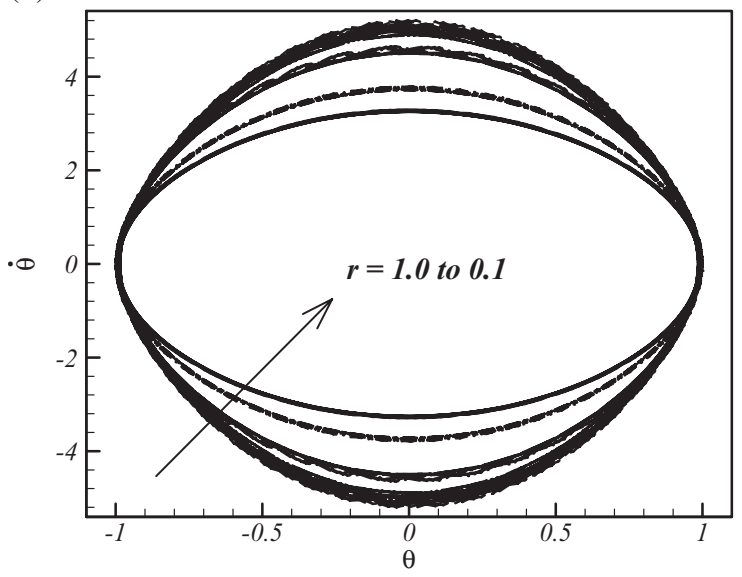

Fig. 5. Phase plane for Example 2; (a): variation of $l$; (b): variation of $r$. 


\section{Conclusions}

In this paper, two methods, Lagrange and HPM, were simultaneously used to obtain an approximate analytical solution for nonlinear problems in dynamics. It was shown that by choosing an appropriate linear operator and vanishing the secular terms, just two orders in series approximation is sufficient to obtain an accurate solution that is valid for the whole domain of the solution. Some

[1] R. L. Burden and J. D. Faires, Numerical Analysis, PWS Publishing Company, Boston 1993.

[2] K. Al-Khaled, S. Momani, and A. Alawneh, Appl. Math. Comput. 171, 281 (2005).

[3] A. S. Mahmood, L. Casasus, and W. Al-Hayani, Phys. Lett. A 357, 306 (2006).

[4] C. S. Gardner, J. M. Green, M. D. Kruskal, and R. M. Miura, Phys. Rev. Lett. 19, 1095 (1967).

[5] A. Kimiaeifar, A. R. Saidi, G. H. Bagheri, M. Rahimpour, and D. G. Domairry, Chaos Solit. Fract. 42, 2660 (2009).

[6] M. Moghimi, A. Kimiaeifar, M. Rahimpour, and G. H. Bagheri, J. Mech. Eng. Sci. 224, 1193 (2010). remarkable virtues of the introduced method were illustrated and their applications to obtain the higher-order approximate periodic solutions of nonlinear problems were demonstrated. The HPM results had a good agreement with those obtained using the numerical method. In addition, it was shown that this method can predict the response of the system and its stability based on the input physical parameters, and HPM is effective and convenient to solve holonomic problems.

[7] M. M. Mousa and A. Kaltayev, Z. Naturforsch. 64a, 788 (2009).

[8] J. H. He, Int. J. Mod. Phys. 20, 1141 (2006).

[9] J. H. He, Int. J. Nonlin. Sci. Numer. Simul. 2, 257 (2001).

[10] J. H. He, Int. J. Nonlin. Mech. 37, 309 (2002).

[11] E. Hesameddini and H. Latifizadeh, Int. J. Nonlin. Sci. Numer. Simul. 10, 1389 (2009).

[12] J. H. He, Int. J. Nonlin. Sci. Numer. Simul. 6, 207 (2005).

[13] J. H. He, Int. J. Mod. Phys. B 20, 2561 (2006).

[14] M. S. Shadloo and A. Kimiaeifar, J. Mech. Eng. Sci. 225, 347 (2011). 